\title{
Abstracts of Theses Approved for the MSc Degrees at the Faculty of Medicine, Health Sciences Centre, Kuwait University, Kuwait
}

\author{
1 \\ Does Added Value of Whole Body Blood \\ Pool Imaging Justify Its Routine Use in Bone \\ Scintigraphy? \\ Abdulredha A.H. Esmail
}

Department of Nuclear Medicine, Faculty of Medicine, Kuwait University, Kuwait

Bone scintigraphy (BS) is a sensitive modality to detect bony abnormalities. The most widely used technique is the 3-phase: flow, blood pool and delayed phases. The blood pool phase shows the leakage of ${ }^{99 \mathrm{~m}} \mathrm{Tc}-\mathrm{MDP}$. Routine limited acquired blood pool images have been helpful in the diagnosis of infection, other pathologies such as trauma, and diagnosis of chronic low back pain. Whole body images of the blood pool can be obtained instead of spot images which are restricted to the region of interest. The objective of this study was to assess the impact of the routine acquisition of whole body blood pool (WBBP) images on the diagnostic potential of multiphase bone scintigraphy in bone diseases and additional soft tissue information. This information could be missed if whole body blood pool images were not obtained, as the routine practice. Over a 1 year period, 608 patients were referred for BS. 474 patients had routine WBBP images acquired within 10 min postinjection. WBBP images were considered useful if it detected findings in regions other than the area of interest. WBBP was found to be useful in $50 \%$ of 474 patients. Findings of significance included extraosseous observations such as breast hyperemia and organ abnormalities. Additionally, skeletal findings were noted which included the determination of the status of joint diseases, determining the age of the fracture and surgical sites whether old or recent. Other findings were noticed in regions away from the region of interest; which were explained by WBBP, assessment of the maturity of heterotopic bone formation and early radiotracer localization at metastatic sites. The data showed that WBBP images were useful to demonstrate many osseous and extraosseous findings and have added value that justify the routine use of this simple option.

Abdelhamid H. Elgazzar (Supervisor)

Gaber Ziada (Co-Supervisor)

2

Scintigraphic Evaluation of Pulmonary Embolism among In-Patients and Out-Patients and Reliability of Standard Criteria for Scintigraphic Interpretation of Early Possible Pulmonary Embolism

\section{Ohoud Rasheed AlKazemi}

Department of Nuclear Medicine, Faculty of Medicine, Kuwait University, Kuwait

Pulmonary embolism (PE) is a potentially fatal and common condition involving hospitalized patients, and often missed clinically because of nonspecificity of symptoms and signs. The objective of this study is to evaluate the characteristics and scintigraphic pattern for ventilation perfusion (V/Q) lung scans for suspected PE in hospitalized patients as compared to out-patients, and whether ventilation perfusion lung scans performed early on within $24 \mathrm{~h}$ of onset of symptoms suggestive of PE provide reliable findings given the transient bronchoconstriction known to occur early after pulmonary embolism. This is a prospective consecutive case study conducted over a three year period among 264 inpatients (age 6-92), and 116 out-patients (age 16-77) referred to V/Q lung scans to exclude PE. Patients presenting within $24 \mathrm{~h}$ following onset of symptoms suggestive of PE with matching venti-

\section{KARGER}

Fax +4161306 1234

E-Mail karger@karger.ch

www.karger.com
(C) 2010 S. Karger AG, Basel

1011-7571/10/0196-0505\$26.00/0

Accessible online at:

www.karger.com/mpp 
lation and perfusion defects had a follow-up lung scintigraphy after $48 \mathrm{~h}$ for comparison. Comparison between hospitalized patients and out-patients showed that the in-patient group was admitted to hospital mostly for a condition un-related to pulmonary thromboembolic diseases, they had more co-morbid illnesses and more underlying risk factors. Also, in-patients were referred earlier to do lung scan after onset of symptoms suggestive of PE and were found to have more abnormal lung scans. There was no relationship between the time of onset of symptoms suggestive of $\mathrm{PE}$ and the lung scan probability. A subgroup of 15 patients who had their lung scan done within $24 \mathrm{~h}$ of their onset of symptoms suggestive of PE with matching defects on ventilation perfusion scans had no significance changes on $48 \mathrm{~h}$ follow-up lung scan after enough time for normalization of presumed ventilation abnormalities associated with emboli. The study showed no significant differences in the ventilation perfusion lung scan patterns between in-patient and out-patient groups. There was no change in the interpretation of V/Q scans done within $24 \mathrm{~h}$ and follow-up lung scans in the small subset of patients.

Abdelhamid H. Elgazzar (Supervisor)

Gaber Ziada (Co-Supervisor)

\section{3}

\section{Semiquantitative Methods for Characterization of Breast Lesions on ${ }^{99 m}$ Tc-Sestamibi Scintigraphy}

Jawaher Abdulhadi Al-Ajmi

Department of Nuclear Medicine, Faculty of Medicine, Kuwait University, Kuwait

Breast cancer is the commonest malignant neoplasm with the highest mortality in women. Mammography is currently the most effective screening modality for detecting early cancer which is not clinically palpable. However, it has limited sensitivity in patients with dense breasts or patients who have implants or surgical intervention. Scintimammography (SM) has been recently found useful in such cases. The aim of our study is to optimize the performance of SM using semiquantitative methods thereby improving its sensitivity and specificity for differentiation of benign from malignant breast lesions. This was a prospective study over 6 months on a group of patients who had equivocal findings on mammography performed for suspicion of a breast mass. SM was done 20 min after i.v. injection of $25 \mathrm{mCi}$ of technetium-99m sestamibi in anterior, lateral and posterior oblique views of torso. The images were analyzed using a standard and a scaled visual system by alteration of the intensity of the image display. Semiquantitation was done by BiLinear Interpolative Background Subtraction (BLIBS) to improve lesion detectability and contrast. Comparison was done with histo-/cytology as a reference test. A total of 69 masses were included, 45 masses had histo-/cytological diagnosis. The sensitivity, specificity, positive and negative predictive values for malignancy using standard visual analysis was: $73,57,56$ and $75 \%$, respectively. The sensitivity increased to $79 \%$ using BLIBS, however, at the expense of reduced specificity, positive and negative predictive values $(42,50$, and $44 \%$, respectively).
We concluded that the use of the semiquantitative approach for analysis of SM was of some benefit in giving an objective means of characterization of breast lesions over the nonquantitative method. The application of such methods for analysis would provide a valuable addition to the overall diagnostic performance of SM.

\author{
Issa Loutfi (Supervisor) \\ Renu Gupta (Co-Supervisor)
}

\section{4 \\ Role of Gallium-67 Citrate Imaging in Graves' Ophthalmopathy}

Asma Ibrahim Al-Shatty

Department of Nuclear Medicine, Faculty of Medicine, Kuwait University, Kuwait

Graves' ophthalmopathy (GO) is a serious inflammatory condition of the eye in Graves' disease. GO is characterized by an early active congestive phase, requiring aggressive anti-inflammatory therapy and a fibrotic phase, that may need surgical management. The objective of this study was to evaluate gallium-67 citrate imaging as indicator of disease activity in GO to predict at an early stage the severity of eye involvement in view of institution of effective treatment. 25 subjects with Graves' disease presenting with mild-to-severe GO and 10 controls with no history of thyroid eye disease were included. GO was classified according to the Clinical Activity Eye Score (CAS) based on 7 test items related to presence of inflammation, such that a CAS of 4 or more denotes activity. Ga-67 citrate, commonly applied as tracer for inflammation, was used to monitor any degree of eye involvement. Imaging involved single photon emission computed tomography (SPECT) of the head at 24 and $72 \mathrm{~h}$ after intravenous injection of $4-6 \mathrm{mCi}$ Ga-67. Analysis included drawing regions of interest around the orbits and temporal-occipital bone on a reframed trans-axial slice. The maximum count ratios in orbit/skull bone $(\mathrm{O} / \mathrm{B})$ were calculated after correction for background activity. CAS showed 5 clinically active GO subjects. Visual inspection of $24 \mathrm{~h}$ images showed intense tracer uptake in the orbits in the affected subjects. Quantitative analysis performed on 35 subjects showed the median o/ $\mathrm{B}$ ratio in the $24 \mathrm{~h}$ images to be 1.7 in GO and 1.3 in controls. The interquartile range (IQR) was 1.4-2.1 for GO and 1.11.6 for controls. The data for the 72-hour images revealed a median of 1.5 for GO and 1.4 for controls. The IQR was 1.3-1.9 for GO and 1.2-1.6 for controls. The O/B ratio for the $24 \mathrm{~h}$ images were significantly different from the $72 \mathrm{hr}$ images ((Mann-Whitney $\mathrm{U}$ test; $\mathrm{p}=0.001)$. Orbital Ga-67 citrate imaging is a sensitive method for predicting early inflammatory eye involvement. The use of the 24-hour imaging time and uptake ratio semiquantitative analysis method would enable to confirm eye pathology. A differential uptake ratio equal or above 1.8 would be considered as a case of Graves' ophthalmopathy. Compared to clinical evaluation by CES, Ga-67 scan would precede clinical manifestation of activity.

Issa Loutfi (Supervisor)

Suhail Doi (Co-Supervisor) 
5

\section{Genetic Aberrations Associated with Diffuse Large B Cell Lymphoma in Relation to Epstein Barr Virus Status}

\section{Aisha Sulaiman Al-Qallaf}

Department of Pathology, Faculty of Medicine, Kuwait University, Kuwait

Diffuse large B cell lymphoma (DLBCL) is a malignant transformation of B-lymphocytes. Worldwide, it represents $40 \%$ of adult non-Hodgkin's lymphoma (NHL). DLBCL is clinically, morphologically and genetically a heterogeneous group of neoplasms, which arise in lymph nodes and/or extranodal sites. Several etiological factors have been implicated in the pathogenesis of lymphoid malignancies including viruses. Epstein-Barr virus (EBV) is a potent $B$ cell-transforming herpes virus that is implicated in the pathogenesis of several tumors including some types of lymphoid malignancies. It is detected in $12 \%$ of sporadic DLBCL, and might contribute to the pathogenesis and prognosis of the disease. In this retrospective study, we studied the prevalence of EBV in sporadic DLBCL cases using three different techniques, which are immunohistochemical staining (IHC) for the detection of EBV latent membrane protein-1 (LMP-1), in situ hybridization (ISH) for the detection of EBV-encoded RNAs (EBER), and nested-PCR (n-PCR) for EBV genotyping. EBV was positive in $6.97 \%$ using IHC, $11.62 \%$ using ISH, and $25.6 \%$ using n-PCR, which was more sensitive in detecting EBV than IHC and ISH. The overall EBV detection rate was $32.5 \%$. EBV-negative $\left(\mathrm{EBV}^{-}\right)$DLBCL cases showed BCL6 protein expression more frequently than EBV-positive $\left(\mathrm{EBV}^{+}\right) \mathrm{DLBCL}$ cases. Other significant findings in the study include a lower median age than what is generally reported in the literature (46 years) with male preponderance. The frequency of chromosomal aberrations in DLBCL cases among Kuwaiti patients is higher compared to non-Kuwaiti patients. All cases showed genetic aberrations (gains/losses), with amplifications being significantly higher than deletions. Furthermore, genetic aberrations were significantly more frequent in extranodal DLBCL cases. Also, some chromosomal alterations were significantly higher in extranodal compared to nodal DLBCL cases including chromosomal amplification of $1 \mathrm{p}, 7 \mathrm{p}, 22 \mathrm{q}, \mathrm{Xp}$ and Xq; and chromosomal deletions of $4 \mathrm{p}, 4 \mathrm{q}$ and $6 \mathrm{q}$. Moreover, BCL2 was expressed in $51 \%$ of our DLBCL cases, with this expression correlating significantly with $b c l 2$ gene amplification and with nodal disease. CD10 was significantly associated with extranodal site of involvement, and CD10 ${ }^{+}$ DLBCL had frequent amplification of $7 \mathrm{q}$ compared to $\mathrm{CD} 10^{-}$ DLBCL cases. BCL6 and CD10 protein were also significantly associated, and CGH resulted in some genetic distinctions among $\mathrm{BCL}^{+} / \mathrm{CD} 10^{+}$and $\mathrm{BCL} 6^{-} / \mathrm{CD} 10^{-}$DLBCL cases. In conclusion, DLBCL is a markedly heterogeneous disease. The reported prevalence of $\mathrm{EBV}^{+} \mathrm{DLBCL}$ cases in this study is higher than what is reported in the literature, especially when n-PCR technique was used. There were some significant differences between $\mathrm{EBV}^{+}$ DLBCL and $\mathrm{EBV}^{-}$DLBCL cases. The reported phenotypic and genetic findings in this study may be related and contribute to the pathogenesis and prognosis of DLBCL. Further studies correlating these findings with clinical features are warranted.

Salah A. Al-Humood (Supervisor)

Fahd Al-Mulla (Co-Supervisor)

\section{6}

A Comprehensive Study on Whole Genome Amplification of DNA

Mira Ali Bosso

Department of Pathology, Faculty of Medicine, Kuwait University, Kuwait

The availability of sufficient quantity and quality of DNA is crucial to any genetic analysis, especially with the current genomewide analytical tools that require high DNA input such as single nucleotide polymorphism (SNP) platforms and microarray technology. Whole genome amplification (WGA) methods were developed in an attempt to immortalize DNA resources through multiplying nanogram amounts of DNA to give microgram quantities that are adequate for multi-stage genetic testing. In this study we evaluated the ability of a newly modified GenomePlex ${ }^{\circledR}$ methodology to representatively amplify challenging, partially degraded DNA samples without introducing bias in the original composition of DNA. Tissue obtained from microdissection of deparaffinized formalin-fixed, paraffin-embedded microscopic sections were mildly and randomly fragmented and GenomePlex DNA amplification was performed. The purified amplified products were assessed by gel electrophoresis, spectrophotometer measurement and real-time quantifier assay. Pairs of WGA amplified samples and their un-amplified counterparts were examined by several different genotyping methods and the results were comparatively analyzed. Results obtained from both metaphase and array based comparative genomic hybridization demonstrated excellent genome coverage by the GenomePlex technique and accurate representation of genome wide copy number profile with insignificant errors observed. Real-time relative quantitation investigation of targeted exonic and intronic dosages in GenomePlex amplified samples showed significant discrepancies from the dosages found in the pre-amplified stage with a trend toward overrepresentation in the post amplified states. It is therefore recommended to avoid the use of amplified samples in real-time relative quantitation assays. Further analysis showed that GenomePlex method was able to accurately reproduce a point mutation present in MSH2 Exon2 (226 C>T) in the WGA amplified DNA extracted from hereditary non-polyposis colon cancer (HNPCC) representative blood samples. This mutation was confirmed by sequencing and the GenomePlex technique introduced no additional mutations to the original sequence. GenomePlex investigation by real-time allelic discrimination assay on two SNPs (rs2088702 and rs904661) showed that $92.8 \%$ and $82.14 \%$ of tested formalin-fixed, paraffin-embedded (FFPE) samples maintained SNP allelic ratios, respectively. Genomewide GeneChip ${ }^{\circledR}$ analysis of 10,000 SNPs in before and after GenomePlex ${ }^{\circledR}$ amplified samples of high-quality DNA showed $97.9 \%$ concordance in SNP profiles demonstrating the high accuracy of the GenomePlex technique. GeneChip analysis of FFPE sample showed $80 \%$ concordance in SNP profile of before and after GenomePlex amplification. Our results demonstrate the ability of the GenomePlex technique to amplify DNA of FFPE tissue and maintain the genome wide copy number and allelic ratio evident from comparative genomic hybridization $(\mathrm{CGH})$, array $\mathrm{CGH}$ and GeneChip single nucleotide polymorphism (SNP) analyses, but the technique was not equally reliable in real-time relative quantitation 
assays. Such confirmation has a great impact on research and offers an opportunity to rescue precious samples and provide unlimited supply of DNA banks.

Fahd Al-Mulla (Supervisor)

Olusegun Mojiminiyi (Co-Supervisor)

\section{7}

\section{Effect of Streptozotocin-Induced Diabetes on the Physical Integrity of the Blood-Cerebrospinal Fluid Barrier in Rat}

Fatemah Salem Abdullrida Safar

Department of Physiology, Faculty of Medicine, Kuwait University, Kuwait

Aims/Hypothesis: It has been shown that streptozotocin (STZ) induced diabetes in rats caused an increase in paracellular permeability across the blood-brain barrier (BBB) and altered expression of the BBB tight junction (TJ) proteins. This study investigated effects of the STZ-induced diabetes in rat on the expression of the TJ proteins in the choroid plexus epithelium (CPE), as well as on the physical integrity of blood-cerebrospinal fluid barrier (BCSFB). Materials and Methods: Diabetes was induced in Sprague-Dawley rats by an i.p. injection of STZ, $55 \mathrm{mg} / \mathrm{kg}$; control animals received a vehicle injection. Each group was further divided into the sub-groups, which were used for the study 7 and 28 days after the treatment, respectively. The expression of TJ proteins occludin, claudin 1 and 2 was explored at the transcript level by the quantitative real-time PCR; the fold change in the diabetic groups was calculated using the delta delta Ct method. The expression of TJ proteins at the protein level was explored by immunoblotting; the $\mathrm{CSF} /$ perfusate ratio for $\left[{ }^{14} \mathrm{C}\right]$ sucrose after in situ brain perfusion and the albumin CSF/serum ratio in vivo were estimated to assess the physical integrity of the BCSFB. Results: The amount of mRNA for occludin diabetic animals after 7 days of treatment decreased to $64 \%$ of that in the control; however, no significant change in the amount of mRNA for claudin 1 and 2 was observed. After 28 days of diabetes a decrease in the mRNA amount for claudin 1, claudin 2 and occludin $(47,14$ and $43 \%$, respectively) was observed compared to the control group. No significant change in claudin 1 and 2 and occludin relative expression at the protein level was detected in either group. Also, neither $\left[{ }^{14} \mathrm{C}\right]$ sucrose nor the albumin CSF/serum ratios differed between the diabetic and control animals. Conclusion: STZ-induced diabetes has caused a decrease in the amount of occludin mRNA after 7 days and a decrease in the amount of mRNA for occludin and the two claudins after 28 days. However, the relative expression of those proteins at the protein level and the physical integrity of the BCSFB did not change in diabetes.

Zoran Redzic (Supervisor)

Hameed Alsarraf (Co-Supervisor)

\section{8}

\section{Rat Placental Beta-Catenin and Cyclin D1 mRNA and Protein Expression: Possible Regulation by Estrogens and Their Receptors during Pregnancy}

Amina Abuzaid

Department of Physiology, Faculty of Medicine, Kuwait University, Kuwait

Estrogen (E) is essential for the initiation and maintenance of pregnancy, but high levels can retard both placental and fetal growth. It has been shown that during pregnancy, at high E levels, estrogen receptor (ER) is downregulated, rending the placenta unresponsive to E. The exact mechanism by which this effect is achieved has not been studied. In breast cancer tissue, reduced expression of ER is associated with increased levels of $\beta$-catenin and cyclin D1 proteins and increased cell proliferation rate. Thus, we hypothesized that downregulation of ER in placental tissue is associated with increased $\beta$-catenin and cyclin D1 allowing the placenta to proliferate. Rat placentae were collected at 16, 19, and 21 days of gestation. The expression of $\beta$-catenin and cyclin D1 at both gene and protein levels was studied using real-time PCR (ReT-PCR) and Western blotting (WB) methodologies, respectively. E levels increased with gestation. There was an increase in placental weight between 16 and 19 days of gestation only. There was a significant decrease in $\beta$-catenin mRNA and protein expression between 16 and 19 days of gestation, and 16 and 21 days of gestation. For cyclin D1 mRNA expression, a significant decrease was detected at 21 days of gestation, while a significant decrease in its protein level was detected at 19 and 21 days. These proteins were localized in both the cytosolic and nuclear fractions. Decreased expression of $\beta$-catenin and cyclin D1 may explain the stable weight of the placenta between 19 and 21 days of gestation. The exact molecular mechanisms behind placental weight changes with progression of pregnancy involve a complex array of genes that may involve those other than $\beta$-catenin and cyclin D1.

Maie Al-Bader (Supervisor)

\section{9 \\ Alpha and Beta Estrogen Receptor Gene and Protein Expression in Breast Cancer Tissue and Coexpression of the MTA1 Gene}

Shorooq Barrak Mezel

Department of Physiology, Faculty of Medicine, Kuwait University, Kuwait

Breast cancer has become a major concern for women today. It is the second most common type of cancer worldwide. Estrogens stimulate cell division of breast cells and they exert their actions on target cells through estrogen receptors, alpha and beta (ER $\alpha$ and ER $\beta$ ). Several mRNA splice variants exist for both receptors and the normal estrogen function results from a balance between the wild-type ERs and their functional variants that may interfere with the coexpressed wild-type forms in a dominant 
negative manner, or by becoming ligand-independently activated. Immunohistochemistry, Western blotting followed by immunodetection, and real-time PCR were performed to detect the expression of ERs in formalin-fixed paraffin-embedded (FFPE) breast cancer samples in order to find a reliable method for the routine assessment of ER status. In addition, the expression of MTA1 and its variant MTA1s in breast cancer tumors was determined. MTA1 is involved in the transcriptional signaling pathways and its variant MTA1s sequesters ERs in the cytoplasm leading to the activation of non-genomic functions of estrogens. Using the three different protocols, ER isoforms were detected at both the mRNA and protein levels, however, the detection of variants was verified using ReT-PCR only. Although smaller molecular weight bands were seen using Western methodology, the specific variant could not be verified. The results indicated the presence of $E R \alpha$ variants, $\Delta 3, \Delta 5, \Delta 6, \Delta 6+7$ and $\Delta 7$, and $E R \beta 1$ isoform and its variants, ER $\beta 2$ and ER $\beta 5$, in several FFPE breast cancer tissues. In addition, MTA1 and MTA1s were found to be expressed in FFPE breast cancer samples. Out of the three methods studied, ReT-PCR was proven to be the most convenient way to assess the status of ER isoforms and their variants. This method may provide an invaluable tool in the clinical field to predict the response of patients to antiestrogen therapy.

Maie Al-Bader (Supervisor)

\section{0 \\ Molecular Mechanisms of Antiestrogen Regulation of SJSA1 Osteosarcoma Cell Growth}

Sarah Muzahum Al-Awak

Department of Biochemistry, Faculty of Medicine, Kuwait

University, Kuwait

Selective estrogen receptor modulators (SERMs) such as tamoxifen, 4-hydroxytamoxifen and raloxifene and selective estrogen receptor disrupters (SERDs) such as fulvestrant are used in the treatment of human breast cancer and in hormone replacement therapies for postmenopausal women. In addition to their antiestrogenic (antagonist) effects in breast cells, SERMs have estrogenic action (agonist) in the bone. The use of antiestrogens for the treatment of breast cancer might have undesirable side effects such as induction of osteosarcoma whereas the use of SERDs may cause osteopenia. The mechanisms by which SERMs and SERDs modulate the growth of bone cells are still poorly understood. We aimed to analyze the effects of SERMs and SERD on the growth of SJSA-1 osteosarcoma, MDA-MB-231 (estrogen receptor negative) and MCF-7 (estrogen receptor positive) human breast cancer cell lines. The effects of tamoxifen, 4-hydroxytamoxifen, raloxifene and fulvestrant on the growth of SJSA-1, MDA-MB-231 and MCF-7 cell lines were studied in different conditions of serum. Our results show that both SERMs and SERDs had antagonistic effect on cell proliferation of breast cancer cells. More interestingly, SERMs showed two different effects on osteosarcoma growth; in low serum concentration SERMs inhibited (antagonistic), whereas in high serum concentration they stimulated (agonistic) cell growth. This opposite effects of SERMs on SJSA1 cell growth was also confirmed by morphological analysis. Fulvestrant was anatagonistic in both breast cancer and osteosarcoma cell lines in presence of low or high serum levels. Western blot analysis showed that the antagonistic effect on SJSA-1 osteosarcoma cell line was associated with stimulation of phosphorylation of p53 on Ser 15 and the overexpression of PCNA. To our knowledge, this is the first report linking the agonistic effect of SERM in osteosarcoma with the inhibition of p53 activity and PCNA activation. Our study determined the exact cell culture conditions by which the SERMs agonistic and antagonistic effects can be identified. It is well known from the literature that transcriptional control of estrogen receptor is mediated via two regions AF1 (ligand-independent domain) and AF2 (ligand-dependent domain). We suggest that the antagonist effect of SERMs in low serum concentration is due to the absence of growth factors that maintain activity of AF1 and to blockage of AF2 by SERMs binding which resulted in stimulation of $\mathrm{p} 53$ phosphorylation causing cell growth arrest. In high serum concentration, the function of AF1 is being maintained by presence of growth factors which was responsible for the agonistic effect of SERMs, even they are blocking AF2 domain. The presence of growth factors promoted cell growth by inhibiting $\mathrm{p} 53$ activation.

Moussa Alkhalaf (Supervisor)

\section{1}

\section{Epidemiology and Antibiotic Resistant Pattern of Acinetobacter spp. in Kuwait Hospitals}

Muna Ali Al-Hubail

Department of Microbiology, Faculty of Medicine, Kuwait University, Kuwait

The species of Acinetobacter have emerged as important opportunistic nosocomial pathogens. They cause a wide variety of illness in severely ill and immunocompromized patients that can be of lethal consequences. Diseases caused by Acinetobacter include respiratory tract infection, bacteremia, meningitis and urinary tract infection. A. baumannii is the most clinically significant member of this genus and the most predominant species in clinical settings. This bacterium has the capacity to rapidly develop antibiotic resistance and thus, compromises all the drugs that were effective against it. Carbapenem was the drug of choice for the treatment of infections caused by Acinetobacter, until resistance against them became widespread thus limiting the therapeutic alternative for this organism. Carbapenemases, such as metallo- $\beta$-lactamases (MBL) and oxacillinases, mediate resistance to this drug and are being reported all over the world. This study was undertaken to determine the prevalence of carbapenem-resistant Acinetobacter in Kuwait and evaluate their genetic mechanism of resistance. In order to achieve these objectives, 250 consecutive isolates were collected from 8 major hospitals in $\mathrm{Ku}$ wait and their susceptibility to 18 antibiotics determined by the E-test method. Carbapenem-resistant isolates were screened for phenotypic MBL production by disk approximation test (DAT) and confirmed by MBL E-test. Genotypic characterization of the resistance mechanisms was carried by PCR. Pulsed field gel elec- 
trophoresis (PFGE) was performed to determine their clonal relatedness. Out of the 250 isolates, resistance rates to amikacin, amoxicillin-clavulanic acid, ampicillin, cefepime, cefotaxime, ceftazidime, cefuroxime, ciprofloxacin, colistin, gentamicin, imipenem, levofloxacin, meropenem, piperacillin, piperacillin-tazobactam, tigecycline, tetracycline and trimethoprim-sulfamethoxazole were $82.8,89.2,95.6,69.6,83.2,72,92.8,73.2,12,68.4,25.2$, $59.2,37.3,82.8,71.2,57.6,13.6$ and $73.6 \%$, respectively. The overall prevalence of carbapenem-resistant isolates was $37.2 \%$ and the overall prevalence of MBL-producing isolates was 74.2 and $57 \%$ by DAT and MBL Etest, respectively. Sixty-five of the 93 carbapenem-resistant isolates were positive for the different genes tested investigated by PCR; 37 were positive for $b l a_{\mathrm{IMP}-1}, 17 b l a_{\mathrm{VIM}-1}, 43$ $b l a_{\mathrm{VIM}-2}, 24 b l a_{\mathrm{SPM}-1}, 32 b l a_{\mathrm{OXA}-23}$ and $1 b l a_{\mathrm{OXA}-24}$. PFGE demonstrated the dissemination of 3 clones among the different hospitals, while the other 3 were restricted to one particular hospital. In conclusion, resistance to carbapenems has reached unacceptable levels in Kuwait and MBLs as well as oxacillinases are highly prevalent among Acinetobacter spp. in our hospitals. Continuous surveillance and stringent infection control measures are needed to control this dissemination of carbapenem-resistance strains.

Support: Supported by a fellowship from the College of Graduate Studies Project No. YM01/08.

Noura Al-Sweih (Supervisor)

V.O. Rotimi (Co-Supervisor)

\section{2 \\ Identification of Sero-Reactive Peptides Encoded by Five Mycobacterium tuberculosis-Specific Genomic Regions Deleted in the Vaccine Strains of Mycobacterium bovis}

Noora Younis Mohammad Al-Khodari

Department of Microbiology, Faculty of Medicine, Kuwait University, Kuwait

Tuberculosis (TB) is a national, regional and international health problem, which has been declared a 'global emergency' by the World Health Organization. The currently diagnostic reagent against TB is the PPD of Mycobacterium tuberculosis, but it lacks specificity. Thus, there is urgent need to identify new M. tuberculosis antigens for specific diagnosis. The aim of this study was to identify immunodominant seroreactive peptides encoded by five M. tuberculosis-specific genomic regions of difference (RD)1, $\mathrm{RD} 4, \mathrm{RD} 5, \mathrm{RD} 6$ and RD7, and confirm the expression of the respective proteins in M. tuberculosis. A total of 775 peptides (25mers and overlapping with neighboring peptides by 10 aa) corresponding to 39 open reading frames (ORFs) predicted in RD1 (12 ORFs, 220 peptides), RD4 (3 ORFs, 80 peptides), RD5 (5 ORFs, 72 peptides), RD6 (11 ORFs, 236 peptides) and RD7 (8 ORFs, 167 peptides) were tested for antibody reactivity using enzyme-linked immunosorbent assay with up to four batches of pooled sera from $10 \mathrm{~TB}$ patients in each pool, and the peptides showing consistent positivity were further tested with individual sera from $100 \mathrm{~TB}$ patients and 100 healthy subjects. These experiments identified three immunodominant peptides (P24 of RD1ORF9, P17 of
RD6ORF3 and P26 of RD6ORF11) with strong reactivity with sera of TB patients but weak reactivity with sera of healthy subjects, thus suggesting their potential in the diagnosis of TB. The attempts to obtain purified recombinant proteins corresponding to the DNA of above ORFs failed, and therefore antibodies to fulllength proteins could not be raised in rabbits. However, anti-peptide antibodies were successfully raised by using pools of peptides corresponding to each protein, and used to detect their presence in culture filtrate and whole cell lysate of $M$. tuberculosis. The results showed that RD1ORF9/Rv3876, RD6ORF3/Rv1508c and RD6ORF11/Rv1516c were detected in whole cell lysate only, which suggested their nonsecreted nature and expression in $M$. tuberculosis.

Support: Supported by a fellowship from the College of Graduate studies and Kuwait University Research Administration Grant No. YM 08/07.

Abu Salim Mustafa (Supervisor)

Rajaa Al-Attiyah (Co-Supervisor)

\section{3 \\ Evaluation of Por A, the Major Outer Membrane Protein of Campylobacter jejuni, as a Potential Vaccine Candidate against $C$. jejuni Infection \\ Anjum Islam \\ Department of Microbiology, Faculty of Medicine, Kuwait University, Kuwait}

Campylobacter jejuni is a food-borne pathogen and a leading cause of diarrhea in humans worldwide. It is also a cause of travelers' diarrhea. Some patients with C. jejuni infection develop systemic complications and neurological sequelae. The economic burden of C. jejuni infection is considerable. Human campylobacteriosis is an immunizing disease and thus offers the possibility of developing a vaccine to control and prevent the infection. Many groups are working on a vaccine, but the development of a suitable vaccine for use in humans is not yet within sight. There are numerous serotypes of $C$. jejuni and immunity is largely serotypespecific. To develop a vaccine that will impart broad protection will entail targeting common antigens that are shared by all serotypes. The major outer membrane protein (MOMP) of C. jejuni known as PorA $(\sim 45 \mathrm{kDa})$ is one such antigen shared by all serotypes. It mediates adhesion of $C$. jejuni to the gut mucosal surface. Humans recovering from natural C. jejuni infection as well as human volunteers infected with $C$. jejuni mount an immune response to this protein. Therefore, PorA antigen has the potential to be used as a subunit vaccine. A recombinant fusion protein, GST (glutathione $S$ transferase)-PorA prepared from C. jejuni strain C31 was evaluated for protective efficacy against challenge with the homologous strain, $C$. jejuni $\mathrm{C} 31(\mathrm{O}: 6,7)$ and three heterologous strains: 48 (O: 19), 75 (O: 3 ) and $111(\mathrm{O}: 1,44)$ in an adult mouse intestinal colonization model. Initially two doses of the vaccine, $50 \mu \mathrm{g}$ and $300 \mu \mathrm{g}$ were evaluated. The low-dose elicited negligible antibody response and provided poor protection. Therefore, the high dose was used for further studies. Adult $\mathrm{BALB} / \mathrm{c}$ mice $(\mathrm{n}=9$ ) were orally immunized with two doses of 
$300 \mu \mathrm{g}$ GST-PorA mixed with $5 \mu \mathrm{g}$ of the mutant $E$. coli heat-labile enterotoxin (LT R192G) as a mucosal adjuvant, given 7 days apart. Control groups included mice receiving $5 \mu \mathrm{g}$ of LT R192G alone or mice receiving phosphate-buffered saline (PBS) only. Intestinal lavage and blood samples were collected at 14 and 28 days post second vaccine dose, respectively. Antibodies in serum and lavage were measured using ELISA coated with either Sarkosyl-purified MOMP from strain C31 or GST-PorA. Mice were then challenged with homologous and heterologous $C$. jejuni serotypes. Fecal shedding of the challenge bacterium was monitored daily for 9 days and the vaccine efficacy based on its ability to prevent intestinal colonization was computed for the 9-day observation period. The high-dose of GST-PorA (300 $\mu \mathrm{g})$ was effective in eliciting an antigen-specific intestinal lavage and serum antibody response with 2,667 and 1,956 endpoint titers, respectively on ELISA with MOMP antigen. When the assay was done with GSTPorA as the antigen, antibody titers were 3,200 and 1,444, respectively. No or minimal antibody response was seen in LT R192G alone administered mice. Immunization with the high-dose GSTPorA protected against colonization with the homologous and heterologous $C$. jejuni serotypes. The protective efficacy against colonization of the homologous strain, $C$. jejuni C31 (O: 6, 7) was $55 \%(\mathrm{p}<0.001)$. The protective efficacies against colonization of the heterologous strains: C. jejuni 48 (O: 19), 75 (O: 3) and $111(\mathrm{O}$ : $1,44)$ were $43 \%(\mathrm{p}<0.001), 29 \%(\mathrm{p}<0.005)$ and $42 \%(\mathrm{p}<0.001)$, respectively. Immunization with LT R192G alone afforded some degree of protection. This can be explained on the basis of cross reactivity with cholera toxin (and hence related LT) with PorA of C. jejuni. These results indicate that PorA provided significant protection against colonization with homologous as well as heterologous serotypes. Thus, PorA has the potential to be used as a subunit vaccine to achieve broad protection against $C$. jejuni.

Support: Supported by a fellowship from the College of Graduate studies and Kuwait University Research Administration Grant No. YM 06/07.

M.J. Albert (Supervisor)

Raj Raghupathy (Co-Supervisor)

\section{4 \\ Characterization of Community-Acquired Methicillin Resistant Staphylococcus aureus Isolated from Kuwait Hospitals}

Eiman Sarkhoo

Department of Microbiology, Faculty of Medicine, Kuwait University, Kuwait

Community-acquired methicillin resistant S. aureus (CAMRSA) is increasing in many parts of the world. In this study, 135 CA-MRSA were obtained from patients in eight hospitals in $\mathrm{Ku}$ wait between January 2005 and December 2006. They were characterized for their antibiotic resistance, the carriage of virulence associated genes including Panton-Valentine leukocidin (PVL), capsular polysaccharides (cap), accessory gene regulators (agr), staphylococcal enterotoxins (SEs) and toxic-shock syndrome toxin (TSST-1), and typed using coagulase gene typing, pulsed-field gel electrophoresis (PFGE), SCCmec and multilocus sequence typing (MLST) to ascertain their relatedness. Representative isolates were investigated for the genetic location of their resistance determinants. Antibacterial susceptibility testing was performed by the disk diffusion method. MIC was determined with E-test strips. Coagulase gene typing, SCCmec typing, PFGE and MLST were performed according to published methods. PCR was used to screen for the carriage of virulence associated genes. Genetic location of some resistance determinants was investigated in curing and transfer experiments. They were confirmed as CA-MRSA by SCCmec typing. They harbored SCCmec type IV (75.6\%), SCCmec type IVa (8.2\%), SCCmec type IVc (7.4\%) and SCCmec type $\mathrm{V}(8.9 \%)$ genetic elements and belonged to types 2 and $5 \mathrm{ccr}$ complex. They were resistant to ciprofloxacin $(20.7 \%)$, kanamycin (62.2\%), fusidic acid (42.2\%), tetracycline (39.3\%), erythromycin (21.5\%), clindamycin (21.5\%), amikacin (13.3\%), tobramycin (13.3\%), chloramphenicol (2.9\%), gentamicin (5.9\%), trimethoprim (5.9\%), spectinomycin (6.7\%) and mupirocin (6.7\%). They were also resistant to cadmium acetate (82.2\%), mercuric chloride (29.6\%) and ethidium bromide (12.6\%). They were all susceptible to rifampicin, quinupristin-dalfopristin, linezolid, fosfomycin, novobiocin, vancomycin and teicoplanin. Altogether, 109 (80.7\%) of the isolates contained 1-4 plasmids per cell which varied between 1.3 and $36.7 \mathrm{~kb}$ in size. Results of curing experiments suggested that resistance to tetracycline; fusidic acid, cadmium acetate and high-level mupirocin were located on plasmids. However, only a 21-kb plasmid borne resistance to high-level mupirocin was transferred in mobilization experiments. Coagulase gene typing resulted in 13 coagulase types with coagulase type $256(35.6 \%)$ as the most dominant. Although PFGE typing revealed 10 PFGE types; three of them consisting of PFGE type 1 (50.4\%), PFGE type $2(23.7 \%)$ and PFGE type $4(12.6 \%)$ were more common. They belonged to sequence types ST80 (50.4\%), ST30 (23.7\%), ST5 (14.1\%), ST1 (4.4\%), ST6 (3.7\%), ST88 (1.5\%), ST834 (1.5\%), ST8 (0.7\%) and ST950 (0.7\%). Overall, $119(88.1 \%)$ isolates were positive for one of agr I, agr II or agr III. Eighty-five (62.2\%) isolates were agr type III, 25 (17.0\%) were agr type I, nine (8.1\%) isolates were agr type II and none of them was positive for agr type IV. 132 isolates were positive for either capsular type 5 (20.7\%) or 8 (77.3\%). Eightythree isolates $(61.5 \%)$ were positive for PVL, while $52(38.5 \%)$ of them yielded negative results. 103 isolates contained genes for up to four SEs while 32 of them were negative. The majority $(76.3 \%)$ of them harbored sei (63\%), seg (41.5\%), sed (29.6\%), sea (10.4\%), seb $(4.4 \%)$, sec (3.7\%), seh (1.5\%), singly or in combinations. None of them was positive for see. The combinations of seg and sei (22.3\%), and sed and sei (19.4\%) were more common. Only $6.7 \%$ of the CA-MRSA isolates harbored tst. Although they belonged to 10 different sequence types, ST80 and ST30 were the most prevalent clones. Their presence in all eight hospitals shows their continuing expansion in Kuwait hospitals.

Support: Supported by a fellowship from the College of Graduate studies and Kuwait University Research Administration Grant No. YM 05/06.

Edet E. Udo (Supervisor) 
15

\section{Characterization of Group B Streptococcus Isolated from Patients at the Maternity Hospital in Kuwait}

Samar S.S. Boswihi

Department of Microbiology, Faculty of Medicine, Kuwait University, Kuwait

Group B streptococcus (GBS), also known as Streptococcus agalactiae, is the most common cause of neonatal sepsis and meningitis. It also causes diseases in pregnant women, elderly and immunocompromized patients. GBS have nine serotypes which vary in their distribution in different countries. Although the organism remains susceptible to beta-lactam antibiotics, several studies have reported increases in resistance to other antibiotics, especially erythromycin and clindamycin which are used for treating penicillin-allergic patients. The aims of this study were to characterize GBS isolates obtained from patients at the Maternity Hospital in Kuwait for their serotypes, antibiotic susceptibility patterns and carriage of genes for putative virulence factors and resistance determinants. It was also sought to type them using PFGE to determine their genetic relatedness. A total of 154 GBS isolates were collected from 1 July to 31 October 2007. They were obtained from HVS, urine, blood and miscellaneous sources. All isolates were serotyped using rabbit antisera (Denka, Seiken, Japan) according to instructions provided by the manufacturer. Antibiotic susceptibility testing was performed by the disk diffusion method. MIC was determined using E-test and agar dilution method for beta-lactams, tetracycline, minocycline, erythromycin, clindamycin, high-level aminoglycosides (gentamicin, kanamycin, streptomycin), rifampicin, linezolid, chloramphenicol, trimethoprim and ciprofloxacin. PFGE was used to determine the relatedness of the isolates. PCR was used to screen for the carriage of genes for virulence factors and antibiotic resistance determinants. DNA sequencing was performed by the Dye-terminator method with the $3130 x \mathrm{l}$ Genetic Analyzer (Applied Biosystems, USA). Conjugation was performed by the filter mating method. The majority of the isolates belonged to serotype $\mathrm{V}$ (38.3\%) followed by serotype III (19.5\%), serotype Ia (10.4\%), serotype II (10.4\%) and 17 isolates were nontypeable (11\%). Although they were all susceptible to the beta-lactams, MIC determination showed that most of the isolates had higher MIC values for penicillin, cefotaxime $(0.047-0.064 \mu \mathrm{g} / \mathrm{ml})$ and ampicillin (0.064-0.094 $\mu \mathrm{g} / \mathrm{ml})$. Eight isolates expressed reduced susceptibility to penicillin (MIC: $0.125-0.19 \mu \mathrm{g} / \mathrm{ml}$ ). All of the kanamycin-resistant isolates contained $a p h 3$. Majority of the tetracyclineresistant isolates contained tetM (95.7\%). Most of the erythromycin-resistant isolates contained ermB (61.1\%) or ermTR (38.9\%). Only one of the five high-level streptomycin resistant isolates contained ant6. All isolates contained genes for virulence factors. However, $s c p B(88.3 \%), \operatorname{lmb}(88.3 \%)$, bca (57.8\%), and $\operatorname{sod} A(55.8 \%)$ were the most common virulence genes. Most of the isolates contained two or more virulence factor genes. PFGE revealed 11 PFGE patterns and subtypes with the majority belonging to PFGE type 1 and its subtypes (34.4\%). A comparison of PFGE patterns with serotypes showed that $62.7 \%$ of serotype $\mathrm{V}$ isolates belonged to PFGE type 1 and its subtypes, the rest of the serotypes were distributed among different PFGE patterns. Genes for the virulence factors were distributed among all serotypes. No virulence factor was restricted to a single serotype. Genes for antibiotic resistance were distributed among all serotypes. However, ermB and ermTR were mostly found among serotype III. The study has shown a wide distribution in serotypes, antibiotic resistance and carriage of genes for virulence factors in GBS isolated in the Maternity Hospital in Kuwait. The study will serve as bases for further studies in GBS in Kuwait and GCC countries.

Support: Supported by the College of Graduate studies and Kuwait University Research Administration Grant No. YM 13/07.

Edet E. Udo (Supervisor)

Noura Al-Sweih (Co-Supervisor)

\section{6 \\ Antiviral Activity of MxA Protein against Different Types of Human Enteroviruses}

Hayfaa A. Abdulkareem

Department of Microbiology, Faculty of Medicine, Kuwait

University, Kuwait

Human enteroviruses cause a broad spectrum of acute and chronic human diseases including respiratory infections, meningitis, encephalitis, pancreatitis and myocarditis. Up to now, no antiviral agents that are active against enteroviruses are available for clinical use. The type I interferon-induced MxA protein has been shown to inhibit the replication of an enterovirus, Coxsackievirus B4, but not cardioviruses such as encephalomyocarditis virus and mengo virus, all members of the Picornaviridae family. Human enteroviruses consist of more than 60 distinct serotypes against which the antiviral activity of MxA was not yet investigated. The main aim of this study was to investigate the susceptibility of different types of enteroviruses commonly found in $\mathrm{Ku}$ wait to the antiviral activity of MxA protein. The entire MxA open reading frame fragment was subcloned into pcDNA3.1 expression vector using Gateway ${ }^{\circledR}$ technology. Vero cells were then mock-transfected or transfected with the MxA expression vector, and the antiviral activity of MxA protein was assessed against different enterovirus prototypes and one clinical Coxsackievirus B1 (CVB1) isolate. The percentage of inhibition of expression of enteroviral RNA and capsid VP1 protein in MxA-transfected Vero cells was then determined. MxA protein was expressed in $96 \%$ of transfected Vero cells. Following infection of MxA-transfected Vero cells with enteroviruses, the expression of enteroviral RNA was inhibited by up to $99 \%$, and that of VP1 protein by up to $85 \%$. However, there was a difference in the percentage of inhibition of enterovirus replication between the different enterovirus prototypes. The MxA protein was inactive against the clinical CVB1 isolate, and the replication rate of CVB1 isolate in MxA-transfected Vero cells was higher than that in mock-transfected Vero cells. Our results suggest a direct interference of MxA protein with enteroviral replication at transcription level, but with different specificity for enterovirus prototypes and clinical isolates. Genetic sequences of the clinical CVB1 isolate and the CVB1 prototype will eventually provide knowledge about sequences that are associated with resistance to MxA protein.

Support: Supported by Kuwait University Research Administration Grant No. YM 05/07.

Wassim Chehadeh (Supervisor) 


\section{7}

\section{Viral Infection and Diabetes Mellitus}

Horeyah A. Sarkhouh

Department of Microbiology, Faculty of Medicine, Kuwait University, Kuwait

Diabetes mellitus is a chronic condition characterized by hyperglycemia that occurs when the pancreas does not produce enough insulin (type 1 diabetes, T1D) or when the body cannot effectively use the insulin it produces (type 2 diabetes, T2D). Genetic factors are thought to be a major component for the development of diabetes mellitus; however, not all people having one or more of the predisposing genes develop diabetes. This suggests that environmental factors are important in the pathogenesis of diabetes mellitus. As is generally realized, there has been speculation on the possible role of viruses as a risk factor in inducing and/ or precipitating diabetes. In particular, enteroviruses (EV) are currently considered as one of the most probable environmental factor involved in the initiation and/or progression of insulinproducing cell destruction leading to T1D. There is also now a groundswell of data to link hepatitis C virus (HCV) infection with the development of T2D. The main objective of this study was to investigate whether there is an association between viral infections and diabetes mellitus in Kuwait. A total of $217 \mathrm{HCV}$-positive patients, $125 \mathrm{HCV}$-negative patients with liver disease, 188 patients with T2D, 102 patients with T1D, and 250 control subjects have been enrolled for this study. The presence of diabetes was ascertained by using American Diabetes Association guidelines based on fasting plasma glucose measurement. Patients with established diabetes mellitus and those with impaired fasting glucose were categorized together as having glucose intolerance. $\mathrm{HCV}$ infection was assessed by testing for serum HCV-specific antibodies and confirmed by detection of HCV RNA using nested RT-PCR; EV infection was assessed by detection of EV RNA using semi-nested RT-PCR. EV RNA was not detected in the blood of diabetic patients and control subjects. Ten (5.3\%) of 188 T2D patients had evidence of past or ongoing HCV infection compared to $3(1.6 \%)$ of 189 healthy subjects $(\mathrm{p}=0.023)$. Most of HCV RNA detected in T2D patients and control subjects were of genotype 4 . The prevalence of glucose intolerance was significantly higher in $\mathrm{HCV}$-infected patients (42\%) than in non-HCV patients with liver disease $(21.6 \%)(p<0.0001)$. After controlling for gender, age and nationality, $\mathrm{HCV}$-infected patients had a risk for GI two times greater than the one observed in non-HCV patients with liver disease (adjusted OR $=2.2$; 95\% CI 1.2-3.9, $\mathrm{p}=0.008$ ). Multivariate logistic regression analysis showed that older age ( $\geq 50$ years) was an independent predictor of GI (adjusted OR = 3.9; 95\% CI 2.4$6.4, \mathrm{p}<0.0001$ ). The association of GI with obesity, HCV genotype, and anti-HCV drugs was not significant. Unlike non-HCV patients, in the group of HCV-infected patients, the prevalence of GI was significantly higher among patients with histological liver disease $(56 \%)$ versus those with no evidence of liver fibrosis or steatosis $(37.7 \%)(\mathrm{OR}=2.1,95 \% \mathrm{CI} 1.1-3.9, \mathrm{p}=0.023)$. Our observations suggest that the pathogenesis of T2D in HCV infection is unique and differs from the usual pathogenesis of T2D in nonHCV patients. Multiple factors such as ageing and liver disease may contribute to the development of diabetes in patients with chronic hepatitis $\mathrm{C}$.
Support: Supported by a fellowship from the College of Graduate studies and Kuwait University Research Administration Grant No. YM 01/06.

Wassim Chehadeh (Supervisor)

Widad Al-Nakib (Co-Supervisor)

\section{8 \\ Effect of Experimentally-Induced Colitis on the Expression and Function of Muscarinic Receptor Subtypes in the Rat Colon}

\section{Dina Moustafa Jragh}

Department of Pharmacology and Toxicology, Faculty of Medicine, Kuwait University, Kuwait

Inflammatory bowel diseases (IBD) such as Crohn's disease and ulcerative colitis are chronic inflammation disorders affecting the gastrointestinal tract. They are characterized by abdominal pain, diarrhea or constipation and are associated with dysmotility of the colon to receptor agonists. Recently, an increased incidence of IBD in Kuwait and other G.C.C. countries has been reported after the Gulf War in 1991. The study was designed to examine the effect of colitis on the expression and function of muscarinic subtypes present in the colon. Trinitrobenzene sulfonic acid (TNBS)-induced colitis model is a well characterized model of IBD and was used in this study. Inflammation was assessed histologically and also biochemically by measuring myeloperoxidase activity. Results obtained showed that treatment with TNBS resulted in a significant increase in myeloperoxidase activity. There was also a reduced colonic reactivity to carbachol characterized by a reduction in the maximum response, but with no change in the potency of carbachol. Muscarinic receptor antagonist such as atropine (non-selective), pirenzepine (M1-receptor), methoctramine (M2-receptor) and 4-diphenylacetoxy-N-methyl piperidine methiodide (4-DAMP, M3-receptor) produced rightward shifts of carbachol concentration response curves in colon segments from control and TNBS-treated rats. In all cases, there was no reduction in the maximum response to carbachol. There was no significant difference in the potency of 4-DAMP between control and TNBS-treated tissues indicating no change in the affinities of M3-receptor subtypes in inflamed tissues. However, methoctramine was significantly more potent in colon segments from TNBS-treated rats indicating increased function of M2-receptors in the inflamed tissues. The level of M1, M2 and M3 proteins were examined using a commercial enhanced chemiluminescence reagent (ECL) Western blot analysis in this study. The results showed that there was a significant increase in the expression of M2 and M3 proteins, whereas the levels of M1 proteins were significantly reduced. It is therefore concluded that the increased expression of muscarinic M2-receptors possibly indicates an increased contribution of muscarinic M2-receptors to carbachol-induced contractions of the inflamed rat colon.

Mabayoje A. Oriowo (Supervisor)

Islam Khan (Co-Supervisor) 